\title{
Practical, highly stereoselective allyl- and crotylsilylation of aldehydes catalyzed by readily available Cinchona alkaloid amide $\uparrow$
}

\section{Received 11th April 2013 \\ Accepted 4th June 2013 \\ DOI: $10.1039 /$ c3sc50973g}

this: Chem Sci, 2013,4,32,

www.rsc.org/chemicalscience

\author{
Yuan Huang, Licheng Yang, Panlin Shao and Yu Zhao*
}

We have demonstrated that bidentate Lewis base catalysts can be constructed based on the Cinchona alkaloid structure that promote highly stereoselective reactions of allyl- and crotyltrichlorosilane with aromatic as well as aliphatic aldehydes (90-99\% ee, $>98 \%$ diastereoselectivity). The catalysts are available in a one-pot procedure in $>70 \%$ yield from cheap starting materials and promote the allylation reactions at ambient temperature. Gram scale reactions with catalyst recovery and reuse showcased the practicality of the catalytic system.

\section{Introduction}

Cinchona alkaloids and their derivatives have played a significant role as a privileged scaffold in asymmetric catalysis. ${ }^{1}$ The strongly basic quinuclidine nitrogen can effectively serve as a ligand for metal catalysis ${ }^{2}$ or as a Brønsted/Lewis base in organocatalytic reactions. ${ }^{3}$ By incorporating into the catalyst structure another $\mathrm{H}$-bond donor $^{4}$ or a metal-based Lewis acid, ${ }^{5}$

(a) known bifunctional catalysis modes:

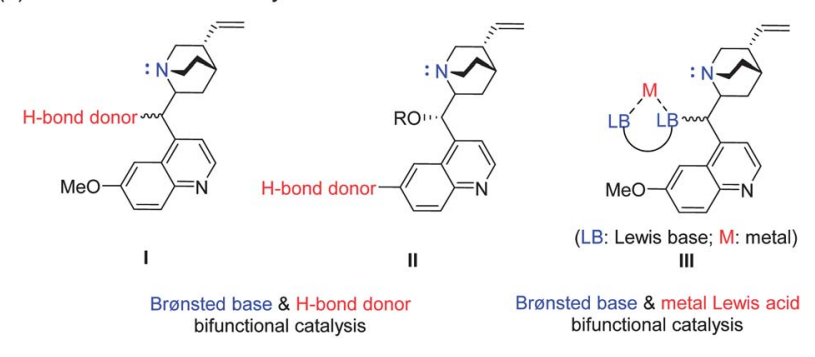

(b) this work:

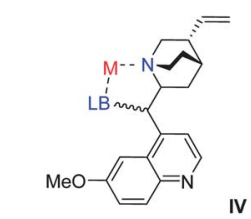

(LB: Lewis base; M: metals, $\mathrm{Si}$, etc.)

bidentate Lewis base catalyst/ligand

Scheme 1 Catalyst design and application. (a) Known catalyst design based on Cinchona alkaloids. (b) Proposed new bidentate Lewis base catalyst.

Department of Chemistry, National University of Singapore, 3 Science Drive 3, 117543, Republic of Singapore. E-mail: zhaoyu@nus.edu.sg

$\uparrow$ Electronic supplementary information (ESI) available: Experimental details, characterization data, and NMR spectral charts. See DOI: 10.1039/c3sc50973g structures such as I, II and III (Scheme 1a) have proven powerful bifunctional catalysts for various organic transformations. Surprisingly, the construction of a simple bidentate ligand or catalyst, employing the quinuclidine nitrogen and another Lewis basic donor moiety (as shown in Scheme 1b), has remained elusive in asymmetric catalysis.

Towards the development of such a catalyst scaffold that will certainly benefit from the readily available, inexpensive Cinchona alkaloids, we chose the addition of allyl- and crotyltrichlorosilane to aldehydes as our model reaction, not only because it is a mechanically well-established reaction that can be catalyzed by a bidentate Lewis base, ${ }^{6}$ but more importantly, it represented the first catalytic approach to realize predictable, diastereospecific crotylation (Type I allylation, ${ }^{6 a}$ where the use of $E$ - or Z-crotylsilane provides anti- or syn-products with $>98 \%$ fidelity through the closed chair transition state), which is a key requirement in the formation of propionate units that are ubiquitous in polyketide natural products. ${ }^{7}$ Many catalytic systems have been developed for this reaction, which are dominantly chiral phosphoramides and $\mathrm{N}$-oxides reported from the groups of Denmark and $\mathrm{Fu}^{,{ }^{6 d}}$ Nakajima et al. ${ }^{6 e f}{ }^{6}$ Malkov and Kočovský et al. ${ }^{6 g, h}$ Hayashi et al. ${ }^{6 i}$ and Snapper and Hoveyda. ${ }^{6 j}$ While the great potential of these methods in chemical synthesis has been demonstrated, ${ }^{8}$ one common limitation is the lack of reactivity for aliphatic aldehydes, with the only exception being the highly stereoselective allyl- and crotylation of aliphatic aldehydes from the Iseki group that required an impractically long reaction time (2-4 weeks) ${ }^{6 k}$ In a related area of research, recent work from the Krische group has revolutionized the field of aldehyde allylation that bypasses the use of allylmetal reagents and can be conducted from either the aldehyde or alcohol oxidation level for both aromatic and aliphatic aldehydes; ${ }^{9}$ by the clever choice of substituted allyl acetates or butadiene, crotylation products with high 
diastereo- and enantioselectivities can also be accessed. ${ }^{\mathbf{1 0}}$ However, considering the diastereospecific nature of Type I allylation, where pure anti- or syn-isomers can be accessed simply based on the choice of crotylating reagent, chiral Type I reagents (mainly allylborations such as Brown allylation, ${ }^{11}$ Roush allylation ${ }^{\mathbf{1 2}}$ and the recent addition of allylsilylation from the Leighton group ${ }^{\mathbf{1 3}}$ ) are still commonly used in asymmetric synthesis; a catalytic Type I allylation that can address the limitations of previous systems is therefore still desired. ${ }^{14}$ Here we present a Cinchona alkaloid amide as a highly efficient and stereoselective catalyst for the allylation and crotylation of a wide range of aldehydes, and in particular, aliphatic aldehydes (95-99\% ee). This system also provides significant practical advantages that enable large scale production: the catalyst can be prepared in a one-pot procedure from inexpensive starting materials, can be easily recovered and reused, and promotes the allylation reactions at ambient temperature (instead of low temperatures of -40 to $-78{ }^{\circ} \mathrm{C}$ for most previous systems).

\section{Results and discussion}

We initiated our studies by examining the catalytic activity of a variety of quinine-derived compounds for the addition of allyltrichlorosilane to 1a, the product of which is highly synthetically useful but was not previously available using Lewis base catalysis (Table 1). Quinine 3 and quinine ester 4 that were previously widely used as nucleophilic catalysts ${ }^{3}$ proved inefficient for our purpose, presumably due to limited Lewis base activation from the monodentate quinuclidine nitrogen (entries 1-2). The well-established bifunctional catalysts ${ }^{4}$ (Brønsted base coupled with $\mathrm{H}$-bond donor) urea 5 and thiourea 6 were also poor catalysts (entries 3 and 4). Sulfonamide 7 (ref. 15) and phosphoric amide $\mathbf{8}$ may serve as bidentate Lewis bases, and interestingly we did obtain product enriched in the opposite enantiomer ( $40 \%$ ee with 7 or 8 vs. $-41 \%$ ee by using 5 ), however the level of efficiency and selectivity were far from satisfactory (entries 5 and 6). To our delight, a simple quinine amide such as $\mathbf{9}$, that has rarely proved successful in asymmetric catalysis, ${ }^{\mathbf{1 6 , 1 7}}$ provided the desired product with high efficiency and excellent enantioselectivity (entry 7). Evaluation of the electronics of the aryl group (entries 7-9) clearly showed that the amide moiety serves as a Lewis base (instead of a $\mathrm{H}$-bond donor in which case the catalyst would be more effective with an electron-withdrawing substituent installed such as 10), with catalyst 11 possessing a strongly electron-donating dimethylamino group being the optimal catalyst ( $88 \%$ conv., 96\% ee). Cinchonidine-derived 12 (only lacking the methoxy substituent on quinoline) provided essentially the same result

Table 1 Optimization of allylation of aliphatic aldehydes ${ }^{a}$

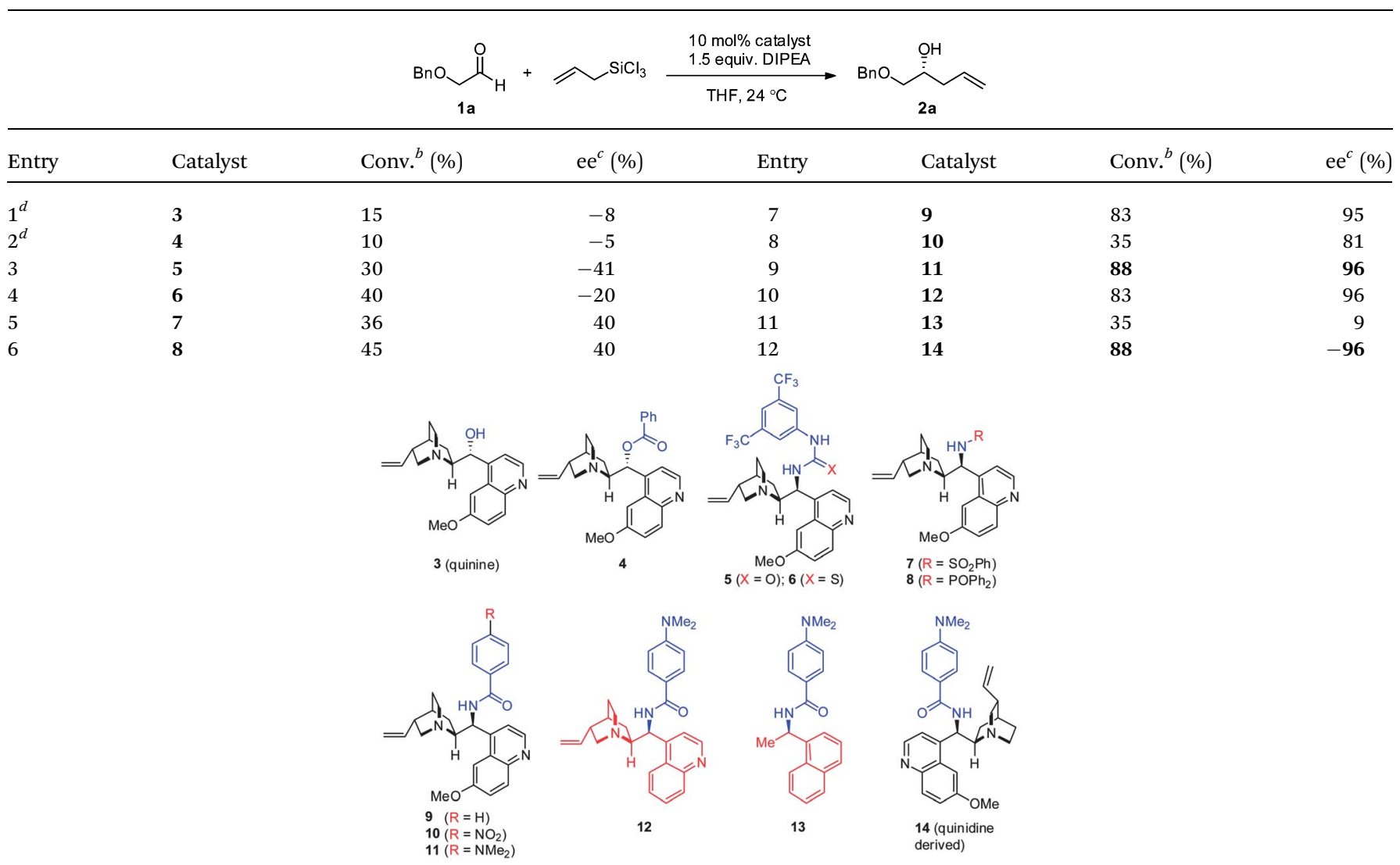

${ }^{a}$ Unless otherwise stated, reactions were run for $24 \mathrm{~h}$. See ESI for details. ${ }^{b}$ Conv. determined by ${ }^{1} \mathrm{H}$ NMR of the crude reaction mixture. ${ }^{c}$ ee determined by HPLC analysis. ${ }^{d}$ Reactions were run for $48 \mathrm{~h}$. 
as 11, suggesting that the quinoline moiety in the catalyst structure is not directly involved in activation of the silylating reagent (entry 10). Compound 13, possessing the analogous chiral amide moiety but lacking quinuclidine, was much less efficient and selective, which further supported our hypothesis of bidentate Lewis base activation of allyltrichlorosilane (entry 11). Finally, as Cinchona alkaloids exist as pseudo-enantiomers, quinidine-derived $\mathbf{1 4}$ was also tested, and provided the enantiomeric product ent-2a with the same excellent enantioselectivity (entry 12).

The evaluation of reaction parameters showed that DIPEA was necessary for the reaction to take place. THF was the optimal solvent in terms of reactivity as well as enantioselectivity. The optimal reaction conditions can be employed to produce a wide range of homoallylic alcohols in excellent enantioselectivity (Table 2). The reactions were carried out with 11 or 14 that yielded both antipodes of the products in comparable excellent enantioselectivity. The enantioselectivities for allylations of aliphatic aldehydes are uniformly high (95-99\%). It is noteworthy that various functional groups such as ethers and silyl ethers (entries 1-3), esters (entry 4) as well as $N$-heterocycles (entry 5) are all well-tolerated, in addition to simple alkyl and alkenyl aldehydes (entries 6-10). This unprecedented scope bodes well for application in complex natural product synthesis. The allylation of $\beta$-chiral aldehyde $\mathbf{1 0}$ was also examined (Scheme 2). While allylation of $\mathbf{1 0}$ (96\% ee) catalyzed by $\mathbf{1 1}$ provided 2o with $98: 2$ diastereomeric ratio, suggesting $>98 \%$ selectivity for the installation of the new stereogenic center, the other diastereomer epi-2o could be obtained with a high diastereomeric ratio of $97.5: 2.5$ from the reaction catalyzed by $\mathbf{1 4}$. Aromatic aldehydes also work under the same conditions to yield products with enantioselectivities ranging between 90 and 94\% ee (entries 11-14, Table 2).

More importantly, we demonstrated that our catalytic system can be applied to the crotylation of aliphatic aldehydes with high enantioselectivity as well as reliable diastereospecificity, characteristic of Type I allylation. As shown in Table 3, with the use of either $E$ - or $Z$-crotyltrichlorosilane 15 (each prepared in one step from commercially available starting materials), ${ }^{18}$ the alcohol products 16 of the two ether-containing substrates were obtained with excellent ee as well as high $\mathrm{dr}$ (>99\% transfer of the geometry of crotylsilane to the product diastereomeric ratio). In contrast, the classical chiral Lewis acid-catalyzed addition of allylic organometallic reagents ( $\mathrm{Si}, \mathrm{Sn}, \mathrm{B}$ ) to aldehydes (Type II allylation; open transition state) provides a mixture of diastereomers, predominantly syn-isomer, independent of starting allylic geometry, while the addition of allylic organometallic reagents $(\mathrm{Cr}, \mathrm{Zn}$, In) generated in situ from the corresponding allylic halides catalyzed by chelating ligands (Type III allylation) yields predominantly the anti-isomer regardless of starting allylic geometry. ${ }^{6 a}$

\section{Practical, scalable allylation and crotylation}

It is noteworthy that the current catalytic system is simple to apply at ambient temperature using a readily available catalyst, and commercial reagents (allyltrichlorosilane, DIPEA, etc.) as
Table 2 Substrate scope for allylation of aldehydes ${ }^{a}$

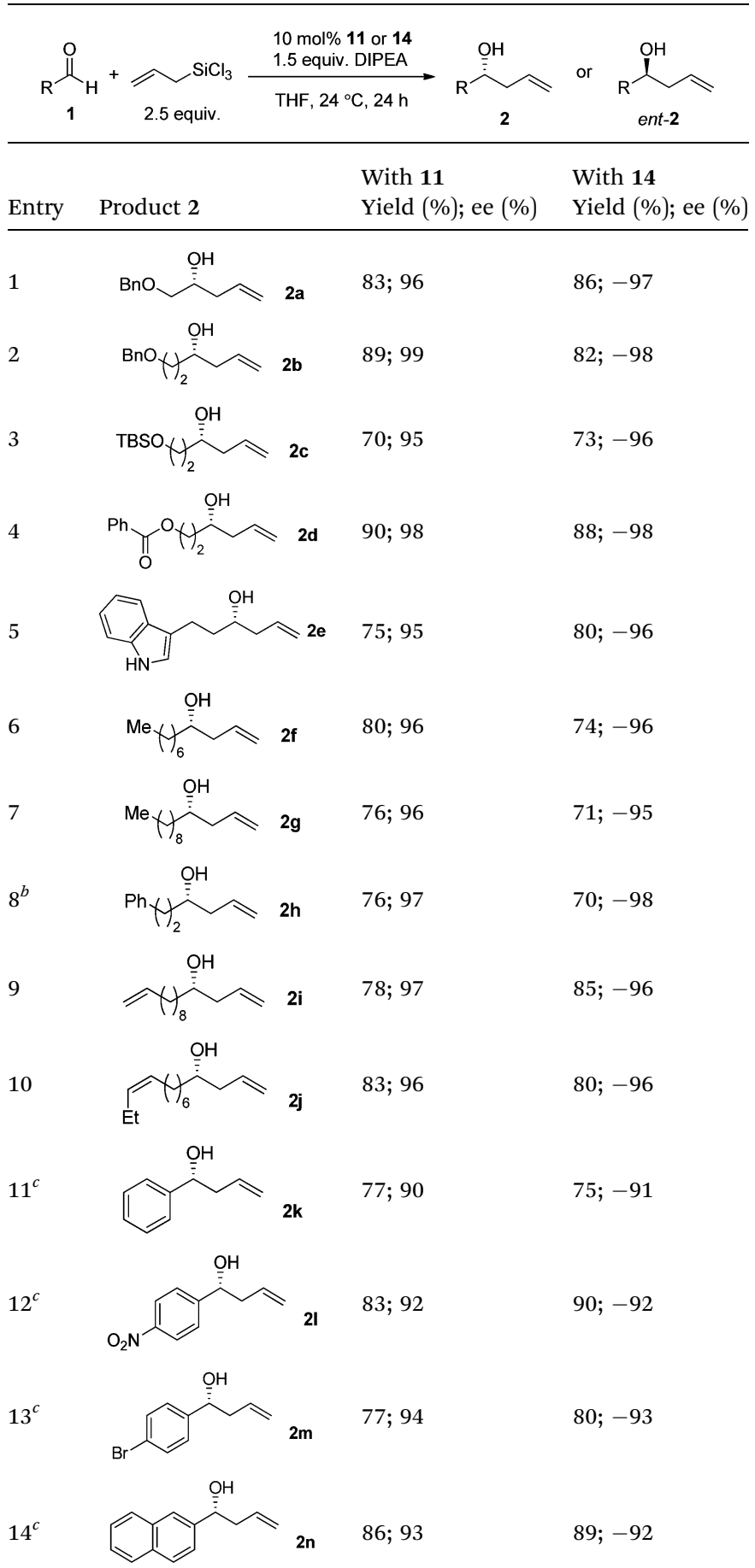

${ }^{a}$ All reactions were carried out at ambient temperature for $24 \mathrm{~h}$. The yields are isolated yields based on the average of two runs. See ESI for details. ${ }^{b} 20 \mathrm{~mol} \%$ catalyst was used. ${ }^{c}$ Toluene was used as the solvent that provided higher conversion for aromatic aldehydes.

received from popular vendors without further purification. As stated earlier, the catalyst can be easily prepared from inexpensive starting materials via a one-pot procedure that includes the previously reported Mitsunobu reaction of quinine with 


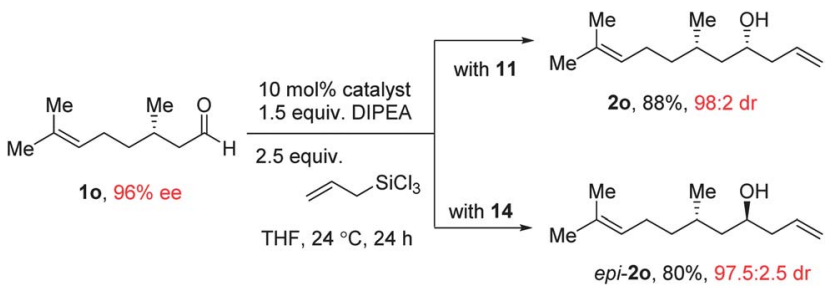

Scheme 2 Allylation of chiral aldehyde.

diphenylphosphoryl azide followed by Staudinger reaction to yield 9-amino-9-deoxy-quinine, ${ }^{19}$ and finally acylation using commercially available 4-(dimethylamino)benzoyl chloride to yield the amide catalyst (Scheme $3 \mathrm{a}$ ). The yield for the one-pot procedure, after a single purification by silica gel chromatography, was over $70 \%$. To further showcase the utility of the system, gram-scale allylation of 1a was carried out that yielded 2a with comparable chemical yield and enantioselectivity to the small scale reactions (Scheme $3 \mathrm{~b} v$ s. Table 2). The selectivity of this system is not sensitive towards concentration or heat transfer (as it is carried out at ambient temperature) so scaling up was straightforward. Although a relatively high catalyst loading of $10 \mathrm{~mol} \%$ is required for the reaction, the catalyst could be easily recovered nearly quantitatively. When the recovered catalyst was used for another gram-scale crotylation of 1a, alcohol 16b was obtained in high diastereo- and enantioselectivity (Scheme 3c).

\section{Mechanistic considerations}

It has been showcased by the Denmark group that a Lewis base can liberate a chloride ion from $\mathrm{SiCl}_{4}$ or allyltrichlorosilane to form a silicate intermediate (17 in Scheme $4 a) .{ }^{20}$ In the case of (a)

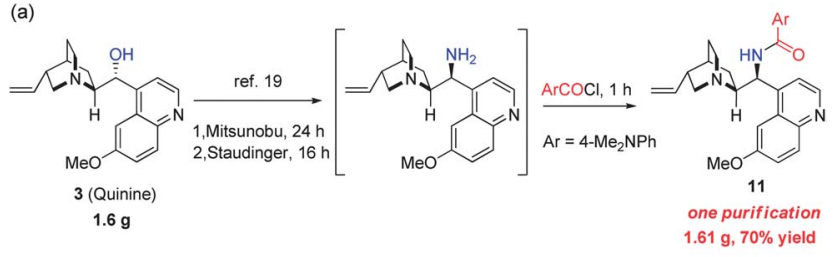

(b)
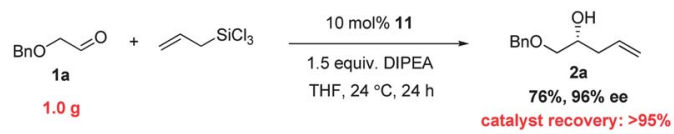

(c)

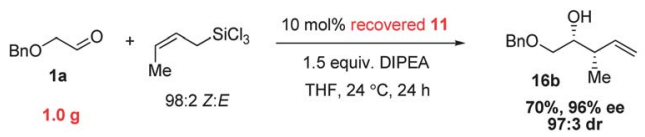

Scheme 3 One-pot catalyst synthesis and gram-scale reactions with catalyst recovery and reuse.

aliphatic aldehydes, however, this chloride adds to the aldehyde to form the corresponding $\alpha$-chloro silyl ether 18 that is presumably responsible for the lack of allylation reactivity for such substrates. In our case, it was also observed that catalyst $\mathbf{1 1}$ binds to $\mathrm{SiCl}_{4}$ to liberate a chloride that quickly adds to aliphatic aldehydes to generate the $\alpha$-chloro silyl ethers $(>60 \%$ conv. in $10 \mathrm{~min})$. On the other hand, the related product was not clearly observed when we mixed $\mathbf{1 1}$ with allyltrichlorosilane and aliphatic aldehyde (Scheme 4). This is in large contrast to the control experiment using HMPA, which promotes this undesired reaction with both $\mathrm{SiCl}_{4}$ and allyltrichlorosilane. This may be due to the relatively lower Lewis basicity of our catalyst compared to HMPA, which is a fortunate character for the success of aliphatic aldehyde allylation.

Table 3 Diastereospecific crotylation of aliphatic aldehydes

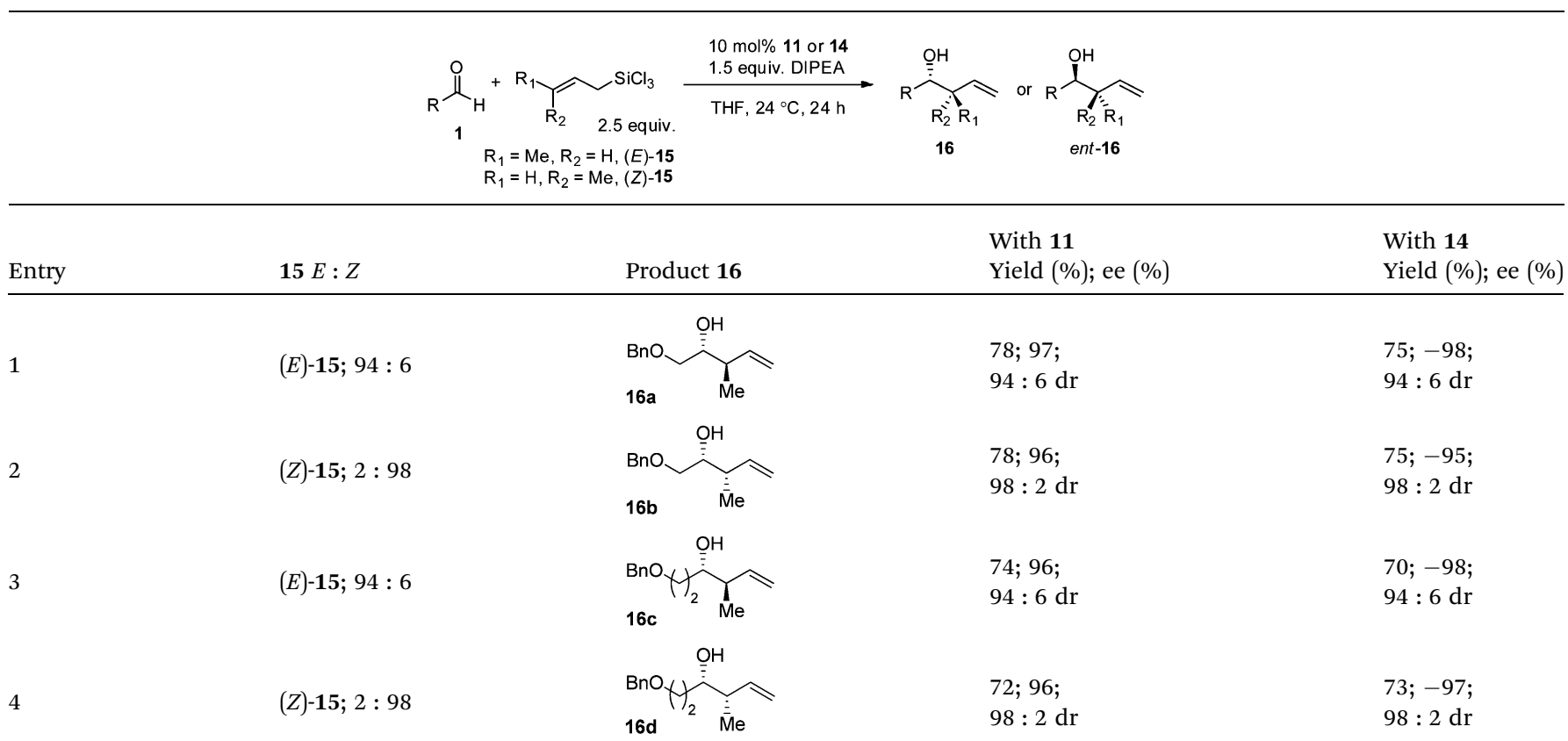

${ }^{a}$ See Table 2 and ESI. 


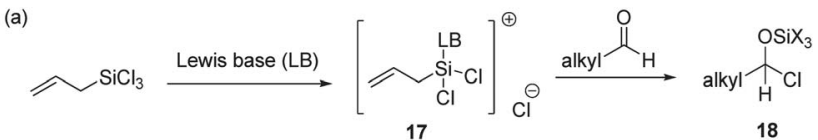
$\angle B=H M P A: \quad \sim 50 \%$ conv. to 18 in $10 \mathrm{~min}$

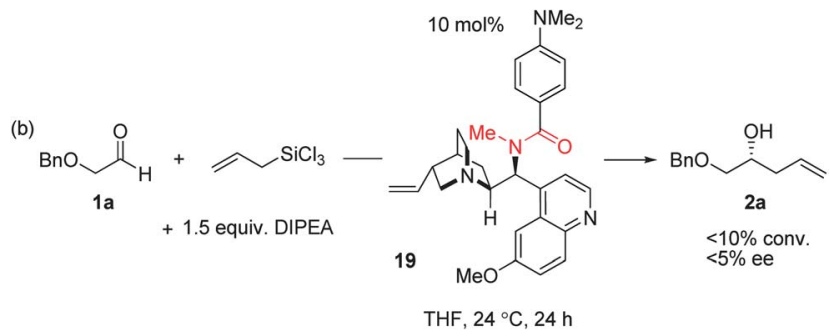

Scheme 4 Exploration of chloride ion liberation and catalyst modification.

It is noteworthy that the secondary amide moiety in our catalyst may react with allyltrichlorosilane in the presence of DIPEA to generate the corresponding $O$-silyl imidate, in which process the chloride ion that is liberated from the silane will be sequestered as part of the DIPEA $\cdot \mathrm{HCl}$ salt (and thus avoid the undesired $\alpha$-chloro silyl ether formation). As stated earlier, DIPEA was found to be essential for the allylation reaction to proceed to high conversion. Preliminary NMR studies by mixing the catalyst, silane, and DIPEA in a $1: 1: 1$ ratio were inconclusive as a complex mixture was formed; however, the resulting mixture was shown to be catalytically active. We have further tested the activity of catalyst $\mathbf{1 9}$ with a methylated amide moiety, which, under otherwise identical conditions, led to only $<10 \%$ conv. to the allylation product with $<5 \%$ ee (Scheme $4 \mathrm{~b}$ ). While the steric hindrance of this catalyst may certainly contribute to this low activity and selectivity, it provides support for the $O$-silyl imidate formation from catalyst 11 and 14. More extensive mechanistic studies as well as calculations will be carried out to further elucidate the nature of the active catalytic species as well as the turnover of the "anionic" catalyst.

The conformational analysis of related Cinchona alkaloid amides both in solution and in the solid state has been performed by the Brunner group using NMR, X-ray as well as molecular orbital calculations during their studies of enantioselective decarboxylation reactions using these catalysts as chiral Brønsted bases. ${ }^{\mathbf{1 6}}$ These studies showed that these molecules prefer the open conformation, ${ }^{21}$ where the quinuclidine nitrogen points away from the quinoline unit, and in turn, towards the 9-amide moiety. In particular, the calculated minimum energy conformation of the protonated cinchonine amide 20 (Scheme 5a) possesses a $\mathrm{H}$-bond interaction between the amide oxygen and the ammonium hydrogen. ${ }^{16 \boldsymbol{b}}$ These data pointed to the possibility of 9-amide and quinuclidine serving as a bidentate catalyst. We have also carried out kinetic studies of our catalytic system, which suggested that the allylation reaction is first-order dependent on the catalyst (see ESI $\dagger$ for details), lending further evidence for the bidentate nature of our catalyst. Based on the above rationale, we propose the transition state model (with catalyst 11) in Scheme $5 b$. (a)

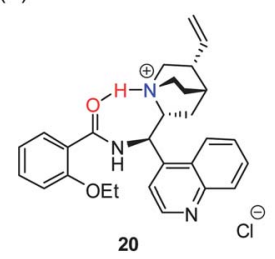

(b) block the top

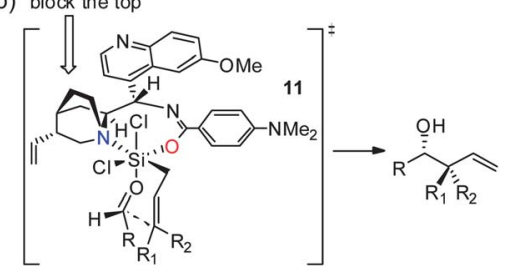

Scheme 5 Proposed reaction transition state model.

On the basis of the principles and mechanistic studies presented by the Denmark group ${ }^{22 a}$ and others, ${ }^{22 b, c}$ the aldehyde was placed trans to chloride to increase its electrophilicity; the allyl group, on the other hand, would coordinate trans to the strongly Lewis basic quinuclidine nitrogen, rendering it more nucleophilic. While the quinoline moiety points away to the back, the quinuclidine moiety effectively blocks the top of the complex so that the aldehyde is placed underneath. Allylation/crotylation through the chair like Zimmerman-Traxler transition state $^{23}$ then provides the desired product in excellent enantioselectivity and predictable, perfect diastereoselectivity.

\section{Conclusions}

In conclusion, we have demonstrated, for the first time, the utility of bidentate Lewis base catalysts constructed from Cinchona alkaloids in the highly stereoselective allyl- and crotylsilylation of aldehydes. The catalytic procedure provides a practical, scalable preparation of various homoallylic alcohols that are useful building blocks in organic synthesis. Current efforts in these laboratories are focused on detailed mechanistic studies to further elucidate the origin of the asymmetric induction, further extending the synthetic utility of the system to allyl/crotylation of chiral aldehydes and application of this family of catalysts to other important organic transformations.

\section{Acknowledgements}

We are grateful for the generous financial support from the Singapore National Research Foundation (NRF Fellowship) and the National University of Singapore.

\section{Notes and references}

1 For selected reviews, see: $(a)$ T. P. Yoon and E. N. Jacobsen, Science, 2003, 299, 1691; (b) K. Kacprzak and J. Gawroñski, Synthesis, 2001, 961; (c) H. Li, Y. Chen and L. Deng, in Privileged Chiral Ligands and Catalysts, ed. Q. Zhou, Wiley, 2011, p. 361.

2 For selected reviews, see: (a) H. C. Kolb, M. S. VanNieuwenhze and K. B. Sharpless, Chem. Rev., 1994, 94, 2483; (b) R. R. Deshmukh, D. H. Ryu and C. E. Song, in Cinchona Alkaloids in Synthesis and Catalysis, ed. C. E. Song, Wiley-VCH, 2009, p. 73. 
3 For selected reviews, see: (a) S.-K. Tian, Y. Chen, J. Hang, L. Tang, P. Mcdaid and L. Deng, Acc. Chem. Res., 2004, 37, 621; (b) C. Palomo, M. Oiarbide and R. Lopez, Chem. Soc. Rev., 2009, 38, 632; (c) T. Marcelli and H. Hiemstra, Synthesis, 2010, 1229; (d) E. M. O. Yeboah, S. O. Yeboah and G. S. Singh, Tetrahedron, 2011, 67, 1725. For pioneering work on Cinchona alkaloid-catalyzed highly enantioselective reaction via Lewis base catalysis, see: $(e)$ S.-K. Tian and L. Deng, J. Am. Chem. Soc., 2001, 123, 6195. 4 S. J. Connon, Chem. Commun., 2008, 2499.

5 L. Stegbauer, F. Sladojevich and D. J. Dixon, Chem. Sci., 2012, 3, 942.

6 For selected reviews, see: (a) S. E. Denmark and J. Fu, Chem. Rev., 2003, 103, 2763; (b) M. Yus, J. C. GonzálezGómez and F. Foubelo, Chem. Rev., 2011, 111, 7774; (c) A. V. Malkov and P. Kočovský, Eur. J. Org. Chem., 2007, 29. For representative examples, see: (d) J. Fu and S. E. Denmark, J. Am. Chem. Soc., 2001, 123, 9488; (e) M. Nakajima, M. Saito, M. Shiro and S. Hashimoto, $J$. Am. Chem. Soc., 1998, 120, 6419; (f) M. Nakajima, S. Kotani, T. Ishizuka and S. Hashimoto, Tetrahedron Lett., 2005, 46, 157; $(g)$ A. V. Malkov, M. Orsini, D. Pernazza, K. W. Muir, V. Langer, P. Meghani and P. Kočovský, Org. Lett., 2002, 4, 1047; (h) A. V. Malkov, L. Dufková, L. Farrugia and P. Kočovský, Angew. Chem., Int. Ed., 2003, 42, 3674; (i) T. Shimada, A. Kina and T. Hayashi, J. Org. Chem., 2003, 68, 6329; (j) J. F. Traverse, Y. Zhao, A. H. Hoveyda and M. L. Snapper, Org. Lett., 2005, 7, 3151; (k) K. Iseki, S. Mizuno, Y. Kuroki and Y. Kolmyashi, Tetrahedron Lett., 1998, 39, 2767. The Zhu group reported a catalytic asymmetric allylation of aliphatic aldehydes, but no crotylation reactions were disclosed, see: (l) B. Bai, L. Shen, J. Ren and H. J. Zhu, Adv. Synth. Catal., 2012, 354, 354.

7 J. Staunton and K. J. Weissman, Nat. Prod. Rep., 2001, 18, 380 .

8 (a) S. E. Denmark and J. Fu, Org. Lett., 2002, 4, 1951; (b) S. E. Denmark, C. S. Regens and T. Kobayashi, J. Am. Chem. Soc., 2007, 129, 2774.

9 For methodology development, see: (a) I. S. Kim, M.-Y. Ngai and M. J. Krische, J. Am. Chem. Soc., 2008, 130, 6340; (b) I. S. Kim, M. -Y. Nagi and M. J. Krische, J. Am. Chem. Soc., 2008, 130, 14891; (c) Y. Lu, I.-S. Kim, A. Hassan, D. J. Del Valle and M. J. Krische, Angew. Chem., Int. Ed., 2009, 48, 5018; (d) A.-M. R. Dechert-Schmitt, D. C. Schmitt and M. J. Krische, Angew. Chem., Int. Ed., 2013, 52, 3195. For selected applications to complex molecule synthesis, see: (e) P. Harsh and G. A. O'Doherty, Tetrahedron, 2009, 65, 5051; (f) S. B. Han, A. Hassan, I. S. Kim and M. J. Krische, J. Am. Chem. Soc., 2010, 132, 15559; (g) Y. Lu, S. K. Woo and M. J. Krische, J. Am. Chem. Soc., 2011, 133, 13876; (h) Y. Feng, X. Jiang and J. K. De Brabander, J. Am. Chem. Soc., 2012, 134, 17083.

10 (a) I. S. Kim, S. B. Han and M. J. Krische, J. Am. Chem. Soc., 2009, 131, 2514; (b) J. R. Zbieg, J. Moran and M. J. Krische, J. Am. Chem. Soc., 2011, 133, 10582; (c) X. Gao, H. Han and M. J. Krische, J. Am. Chem. Soc., 2011, 133, 12795; (d)
X. Gao, Y. J. Zhang and M. J. Krische, Angew. Chem., Int. Ed., 2011, 50, 4173; (e) J. R. Zbieg, E. Yamaguchi, E. L. McInturff and M. J. Krische, Science, 2012, 336, 324; $(f)$ E. L. McInturff, E. Yamaguchi and M. J. Krische, J. Am. Chem. Soc., 2012, 134, 20628.

11 (a) H. C. Brown and P. K. Jadhav, J. Am. Chem. Soc., 1983, 105, 2092; (b) H. C. Brown and K. S. Bhat, J. Am. Chem. Soc., 1986, 108, 293.

12 (a) W. R. Roush, A. E. Walts and L. K. Hoong, J. Am. Chem. Soc., 1985, 107, 8186; (b) W. R. Roush, K. Ando, D. B. Powers, A. D. Palkowitz and R. L. Halterman, J. Am. Chem. Soc., 1990, 112, 6339.

13 (a) J. W. A. Kinnaird, P. Y. Ng, K. Kubota and J. L. Leighton, J. Am. Chem. Soc., 2002, 124, 7920; (b) K. Kubota and J. L. Leighton, Angew. Chem., Int. Ed., 2003, 42, 946; (c) B. M. Hackman, P. J. Lombardi and J. L. Leighton, Org. Lett., 2004, 6, 4375; (d) H. Kim, S. Ho and J. L. Leighton, J. Am. Chem. Soc., 2011, 133, 6517; (e) L. M. Suen, M. L. Steigerwald and J. L. Leighton, Chem. Sci., 2013, 4, 2413.

14 The Hall group reported intriguing Brønsted acid-catalyzed allylboration of aldehydes and developed an asymmetric variant catalyzed by a chiral diol that requires multi-step synthesis together with $\mathrm{SnCl}_{4}$ : (a) V. Rauniyar, H. Zhai and D. G. Hall, J. Am. Chem. Soc., 2008, 130, 8481. The Antilla group reported chiral phosphoric acid-catalyzed allylboration of aldehydes: (b) P. Jain and J. C. Antilla, J. Am. Chem. Soc., 2010, 132, 11884.

15 S. H. Oh, H. S. Rho, J. W. Lee, J. E. Lee, S. H. Youk, J. Chin and C. E. Song, Angew. Chem., Int. Ed., 2008, 47, 7872.

16 (a) H. Brunner and P. Schmidt, Eur. J. Org. Chem., 2000, 2119; (b) H. Brunner, P. Schmidt and M. Prommesberger, Tetrahedron: Asymmetry, 2000, 11, 1501; (c) A. E. Taggi, A. M. Hafez, H. Wack, B. Young, D. Ferraris and T. Lectka, J. Am. Chem. Soc., 2002, 124, 6626.

17 For multifunctional Cinchona alkaloid-based catalysts bearing amide as a linker structure, see: (a) J.-R. Chen, X.-L. An, X.-Y. Zhu, X.-F. Wang and W.-J. Xiao, J. Org. Chem., 2008, 73, 6006; (b) X.-F. Xiong, Z.-J. Jia, W. Du, K. Jiang, T.-Y. Liu and Y.-C. Chen, Chem. Commun., 2009, 6994; (c) Q. Zhu and Y. Lu, Angew. Chem., Int. Ed., 2010, 49, 7753; (d) F. Sladojevich, A. Trabocchi, A. Guarna and D. J. Dixon, J. Am. Chem. Soc., 2011, 133, 1710; (e) F. Zhong, J. Luo, G.-Y. Chen, X. Dou and Y. Lu, J. Am. Chem. Soc., 2012, 134, 10222; (f) M. Hayashi, N. Shiomi, Y. Funahashi and S. Nakamura, J. Am. Chem. Soc., 2012, 134, 19366.

18 M. Kira, T. Hino and H. Sakurai, Tetrahedron Lett., 1989, 30, 1099.

19 V. Benedek, S. Varga, A. Csámpai and T. Soós, Org. Lett., 2005, 7, 1967.

20 (a) S. E. Denmark, T. Wynn and G. L. Beutner, J. Am. Chem. Soc., 2002, 124, 13405; (b) S. E. Denmark and J. Fu, Org. Lett., 2002, 4, 1951.

21 G. D. H. Dijkstra, R. M. Kellogg, H. Wynberg, J. S. Svendsen, I. Marko and K. B. Sharpless, J. Am. Chem. Soc., 1989, 111, 8069. 
22 (a) S. E. Denmark and J. Fu, J. Am. Chem. Soc., 2003, 125, 2208; (b) A. V. Malkov, P. Ramíez-López, L. Biedermannová, L. Rulíšek, L. Dufková, M. Kotara, F. Zhu and P. Kočovský, J. Am. Chem. Soc., 2008, 130, 5341; (c) L. Ducháčková, A. Kadlč́iková, M. Kotara and J. Roithová, J. Am. Chem. Soc., 2010, 132, 12660.

23 H. E. Zimmerman and M. D. Traxler, J. Am. Chem. Soc., 1957, 79, 1920. 